\title{
Evaluate the Effects of Aflatoxins on Agriculture Foods and the Role of Bio-detoxification
}

\section{Waseem Khalid ${ }^{1 *}$, Ayesha Jabeen ${ }^{1}$, Muhammad Rehan ${ }^{2}$, Afifa Aziz ${ }^{1}$, Anam Tariq ${ }^{1}$, Chasheen Fizza ${ }^{1}$, Syed Ali Ur Rehman Siddiqui ${ }^{2}$ and Muhammad Abdul Rahim ${ }^{1}$}

${ }^{1}$ Institute of Home and Food Sciences, Government College University, Faisalabad, Pakistan

${ }^{2}$ Institute of Agricultural Sciences, The Punjab University, Lahore, Pakistan

*Corresponding Author: Waseem Khalid, Institute of Home and Food Sciences, Government College University Faisalabad, Pakistan.
Received: October 28, 2020

Published: November 25, 2020

(C) All rights are reserved by Waseem

Khalid., et al.

\begin{abstract}
Food security is necessary for the purpose of human welfare, for this purpose food safety is applied that provides protection against contamination. Agricultural food plays a vital role in human nutrition which is affected due to aflatoxins. The secondary metabolized called aflatoxins are derived from Aspergillus parasiticus and Aspergillus flavus, these metabolize grow on moist foods and responsible for food spoilage. When humans consumed aflatoxins present food that creates various substances like carcinogenic. For the prevention of aflatoxins in foodstuff, detoxification of aflatoxins is used which can limit toxic substances to an acceptable level in various commodities. The bio-detoxification are specific, efficient and environment friendly in eliminating the possible contamination of aflatoxins in foods.
\end{abstract}

Keywords: Aflatoxins; Carcinogenic; Cereal; Bio-detoxification

\section{Introduction}

Food security, in a true sense, is attained when all the primary pillars of food security such as accessibility, utilization, and sustainability of food are at such level as allow each and every person in the world to have access to wholesome, safe, and nutritious food at an affordable price to sustain a healthy and active lifestyle. The weakening of any of these three important pillars can undermine the food security of the society. Food insecurity is detrimental not only to the overall health and welfare of the society but also to the social, political, and economic sectors of the society. Mycotoxin contamination is known to be the main cause of food insecurity in the world [1].

Aflatoxins, which are known to be the most potent natural carcinogens, are synthesized by fungi of the genus Aspergillus, mostly by Aspergillus parasiticus, and Aspergillus flavus; and seldom by Aspergillus tamari, and Aspergillus nomius. B Aflatoxins are mainly produced by Aspergillus flavus, whereas B and G aflatoxins are the product of Aspergillus parasiticus [2]. Aflatoxins owing to their powerful carcinogenic, teratogenic, and mutagenic activity; have been regarded as a grave threat to human and animal health. International Agency for Research on Cancer (IARC) has classified AFB as a human carcinogen [3].

Aflatoxins are cancerous secondary metabolites produced primarily by Aspergillus flavus and Aspergillus parasiticus in agricultural foodstuff such as peanuts, maize grains, cereals, and animal feeds. Aflatoxins are difuranocoumarin molecules synthesized through the polyketide pathway [4]. Six out of 18 different types of aflatoxins that have been identified are considered important and are designated as $B_{1}, B_{2}, G_{1}, G_{2}, M_{1}$ and $M_{2}$, respectively [5]. These aflatoxin groups exhibit molecular differences. For example, the B-group aflatoxins $\left(\mathrm{B}_{1}\right.$ and $\left.\mathrm{B}_{2}\right)$ have a cyclopentane ring while the G-group $\left(G_{1}\right.$ and $G_{2}$ ) contains the lactone ring [6]. Whereas the B-group aflatoxins exhibit blue fluorescence, the G-group exhibits yellow-green fluorescence under ultraviolet (UV) light, thus 
making the use of fluorescence important in identifying and differentiating between the $B$ and $G$ groups. Aflatoxin $B_{1}$ is the most common [7], and the most widespread in the world and accounts for $75 \%$ of all aflatoxins contamination of food and feeds [8]. Aflatoxins $M_{1}$ and $M_{2}$ are hydroxylated products of aflatoxins $B_{1}$ and $B_{2}$, respectively, and are associated with cow milk upon ingestion of $\mathrm{B}_{1}$ and $\mathrm{B}_{2}$ aflatoxins' contaminated feed. Moreover, once formed from $B_{1}$ and $B_{2}$ forms, aflatoxins $M_{1}$ and $M_{2}$ remain stable during milk processing [9].

Owing to being highly hazardous to human health, documentation for the regulation of Aflatoxin levels in food and feed commodities has recently been carried out. An aflatoxin limit of $20 \mu \mathrm{g} /$ $\mathrm{kg}$ for various food and feed commodities has been determined by the US Food and Drug Administration and Pakistan Standards and Quality Control Authority (PSQCA) [10]. According to EU, total aflatoxin and $\mathrm{AFB}_{1}$ level must not exceed $2 \mu \mathrm{g} / \mathrm{kg}$ and $4 \mu \mathrm{g} /$ $\mathrm{kg}$ respectively when regulated with Maximum Residual Limits (MRLs) in cereals [11]. Lately, Codex Alimentarius Commission, the joint FAO/WHO food standards program, set an aflatoxin limit of $15 \mu \mathrm{g} / \mathrm{kg}$ for various food commodities [12].

\section{Aflatoxins (AFs)}

Aflatoxins are known to be among the hazardous groups of the mycotoxins. It was studied that the aflatoxins are produced by the two different species of the fungus known as Aspergillus flavus and Aspergillus parasiticus [13]. These aflatoxins are secondary metabolites commonly known as mycotoxins and are found throughout the world. They also are found on the living plants and crops e.g. wheat, rice maize and other cereal crops. These fungal spores may prove hazardous to living organisms e.g. plants and animals [14]. According to a report regarding aflatoxins it was observed that the about half of the total species of the Aspergillus parasitica produces mycotoxins [15]. Table 1 represents the categories and fungus types that produce aflatoxins.

\begin{tabular}{|l|c|}
\hline Type of Aflatoxins & Sources of Aflatoxins \\
\hline $\mathrm{B}_{1}$ and $\mathrm{B}_{2}$ & $\begin{array}{c}\text { Aspergillus parasiticus and Aspergillus } \\
\text { flavus }\end{array}$ \\
\hline $\mathrm{G}_{1}$ and $\mathrm{G}_{2}$ & Aspergillus parasiticus \\
\hline $\mathrm{M}_{1}$ & $\begin{array}{c}\text { It is metabolite of AFB } \\
\text { ily found in milk of animals as well as } \\
\text { humans. }\end{array}$ \\
\hline
\end{tabular}

Table 1: Types and sources of aflatoxins. Matumba., et al. [18].
The word "Mycotoxin" is originated from two words, mykes (Greek word for fungus) and toxicum (Latin word for poison) [16]. Aspergillus, Fusarium and Penicillium are three most important genera of mycotoxigenic fungi. AFs (Aspergilus), ochratoxins (Aspergillus and Penicillium) and Trichothecenes and Fumonisins (Fusarium) are the major classes of mycotoxins. The disease caused by mycotoxins exposure is known as mycotoxicosis [17].

About 18 different known categories of AFs such as $\mathrm{AFB}_{1}, \mathrm{AFG}_{1}$, $\mathrm{AFB}_{2}, \mathrm{AFG}_{2}, \mathrm{AFM}_{1}$ and $\mathrm{AFM}_{2}$ were distinguished and reported so far. All these toxins produced by fungi endogenously as secondary metabolites, and among these the big names are $A F B_{1}, A_{1} F_{1}$, $\mathrm{AFB}_{2}, \mathrm{AFG}_{2}$ (Figure 1) which are structurally related to each other. Aflatoxins belong to this group are closely related compounds with little difference in their physical properties and molecular weights (Table 2). These are named as $\mathrm{AFB}_{1}, \mathrm{AFG}_{1}, \mathrm{AFB}_{2}$ and $\mathrm{AFG}_{2}$ due to their fluorescence (blue or green) under ultraviolet (UV) light and relative chromatographic mobility during TLC [20].

\begin{tabular}{|l|c|c|c|}
\hline Aflatoxin & $\begin{array}{c}\text { Molecular } \\
\text { Formula }\end{array}$ & $\begin{array}{c}\text { Molecular } \\
\text { Weight }\end{array}$ & $\begin{array}{c}\text { Melting } \\
\text { Point }\end{array}$ \\
\hline $\mathrm{B}_{1}$ & $\mathrm{C}_{17} \mathrm{H}_{12} \mathrm{O}_{6}$ & 312 & $268-269$ \\
\hline $\mathrm{B}_{2}$ & $\mathrm{C}_{17} \mathrm{H}_{1406}$ & 314 & $268-269$ \\
\hline $\mathrm{G}_{1}$ & $\mathrm{C}_{17} \mathrm{H}_{12} \mathrm{O}_{7}$ & 325 & $244-246$ \\
\hline $\mathrm{G}_{2}$ & $\mathrm{C}_{17} \mathrm{H}_{14} \mathrm{O}_{7}$ & 330 & $237-240$ \\
\hline
\end{tabular}

Table 2: Molecular formula and physical properties of aflatoxins. Prettl., et al. [21].
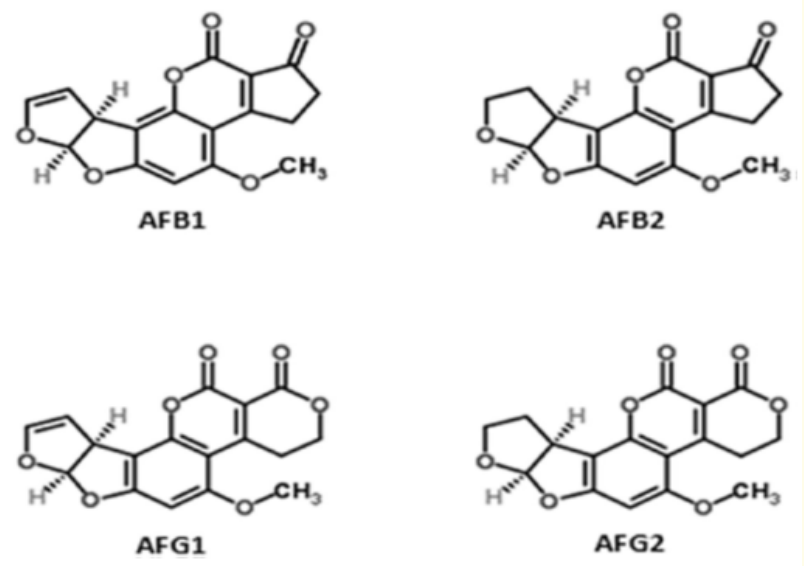

Figure 1: Structure of aflatoxins. Lutfullah., et al. [19]. 
From the historical point of view, aflatoxins were firstly reported in 1960 after the turkey "X" malady which spread as an extreme flare-up in turkey in the United Kingdom and resulted illness and the death of more than 100,000 turkeys alongside other homestead creatures. The main reason for the flare-up was a feedstuff containing Brazilian peanuts that were seriously pervaded by an organism animal varieties Aspergillus flavus. After that flare-up, later examination of the feedstuff revealed a progression of mixes that demonstrates green and blue fluorescent under UV light. These fluorescent mixes were demonstrated as the fundamental driver of Turkey "X" sickness which later known as aflatoxins [22].

The aflatoxins produced by these fungi are favored by the high temperature, moisture and adequate amount of substrate upon which fungus can grow easily. Most probably the fungal pathogens sporoulate well at a temperature range of 24- $27{ }^{\circ} \mathrm{C}$ and a moisture level of more than $13 \%$. It is noteworthy that the fungus of moist and warm regions may attack the wheat kernel during the cropping season. In drought conditions, the chances of aflatoxins productions are more as compared to the normal conditions. The main reason is the low moisture in the soil and high temperature in the environment favored the fungal to sporoulate. In the case of damaged crops due to insects or environmental stress, the fungus can attack easily and destroy the crops more effectively as compared to the healthy plants. The pathogen can attack and harm the post-harvest more easily when stored in improper conditions or damaged at any point of storage. The products become more vulnerable when stored under hot and humid conditions [23].

\section{Legislative limits for aflatoxins}

Due to toxic aspects of aflatoxin international authorities established different control measures and permissible levels. Different measures have been set up for aflatoxins by different countries. Generally, lower tolerance limits established by the industrialized countries as compared to developing countries in which most of the commodities are susceptible. For example, in Malaysia maximum tolerable limit for aflatoxins in all foodstuff is $35 \mu \mathrm{g} / \mathrm{Kg}$, for Sweden MTL is $5 \mu \mathrm{g} / \mathrm{Kg}$ and in Brazil MTL for aflatoxins is $30 \mu \mathrm{g} /$ $\mathrm{Kg}$ [24].

\section{Aflatoxins contamination in agricultural crops}

Cereals are considered as a staple food since ancient times. The grains contain an adequate amount of proteins, fibers and other essential nutrients which are necessary diet for animals and human beings. It has been observed that the grains are more vulner- able to fungal attack during production, harvesting, storage or processing and particularly transportation [25].

Aflatoxin contamination of agricultural produce, owing to its detrimental impacts on public health and its significance in international trade, has gained global attention since last decade. According to the estimates of Food and Agriculture Organization (FAO), mycotoxins are responsible for the contamination of almost $25 \%$ of the agricultural crops every year. Among the most susceptible agricultural commodities are wheat, tree nuts, Maize, Rice, Groundnuts, spices, dried fruits, meat and milk products [26].

It has been observed that $25 \%$ of the crops are affected, directly or indirectly, by fungal diseases every year. Fungi release various types of mycotoxins which are responsible for the contamination and spoilage of food. The food products or foodstuff contaminated with the mycotoxins may harm the humans and animals who consume it and in severe cases leading to the death of human beings. When the food contaminated with the mycotoxins is eaten by the animals, it enters their blood and remains viable for a long period of time and may cause hazardous effects directly or indirectly. In some cases, these mycotoxins are transferred from these animals to their products e.g. eggs, milk, meat and even may also transfer to their offsprings. These products have proved to be harmful to human beings when consumed at any stage of the food chain [7].

The tropical climate is characterized by high rainfall, temperature, and humidity. This type of climate is very much optimum for the growth of fungi. That is why pre and post-harvest losses of agricultural commodities particularly wheat by fungi especially the aflatoxigenic ones in tropical countries are very much evident peculiarly when those commodities have high moisture content either because of delayed drying or any other reason. In Algeria, Aflatoxins contamination in wheat was reported by Riba., et al. [2] in which 108 samples of wheat were collected from two different climatic zones and analyzed by High-Performance Liquid Chromatography. Results showed that $62(56 \%)$ samples were contaminated with AFB1 level up to 37.52ppb.

\section{Rice}

The rice is considered stable food in the world, according to production, its position is 2 nd after wheat. Rice contaminated due to some environmental effects, such as high moisture contents create aflatoxins. Aflatoxin contamination differs from country to country. As aflatoxin B1 is cancer-causing and corresponds to HCC i.e.; he- 
patocellular carcinoma occurs in some countries, therefore a high amount of rice intake on daily basis could be risky [27].

Milled rice grains from Iran were investigated for aflatoxins. It was found that different types of fungi were present on rice grains that contaminated the grains by producing different types of mycotoxins. The continuous growth of rice may not confirm the presence of mycotoxins on the grains, when the samples collected and observed critically the presence of aflatoxins was confirmed with contamination level 20-50ppb. It was greater than the maximum permissible limit of 20ppb [28].

A total of 275 samples of rice and rice products were randomly collected from selected areas of Punjab and assessed for aflatoxins and ochratoxins. Among 275 samples 35\% of the samples were found contaminated with AFs, out of which $19 \%$ and $24 \%$ of samples were found to be above the European Union (EU) maximum content for AFB1 and total AFs, respectively [29].

\section{Wheat}

Khalid., et al. [30] were conducted a study on aflatoxin in wheat flour in which samples were collected from flour markets and flour mills. The moisture level of all samples had less than 14 percent, the higher moisture was seen in the market sample and lower moisture found in flour mills sample. Thin-layer chromatography was used to analyze the aflatoxins (B1, B2, G1 and G2). All markets and mills were free from aflatoxins due to low moisture content.

In Iran a study was conducted by Razdari., et al. [31] in which samples of wheat grains and wheat flour were collected from 7 different provinces of Iran and analyzed for aflatoxin by ELISA method, results showed contamination level up to $14 \mu \mathrm{g} / \mathrm{Kg}$ and $30 \mu \mathrm{g} /$ $\mathrm{Kg}$ respectively.

\section{Maize}

The crucial commodities of agriculture, including maize, cottonseed, peanuts, and pistachio nuts are mostly contaminated by the notorious aflatoxins. Aflatoxins are produced by Aspergillus flavus and related species as secondary metabolites that have the capability of leaving a bad impact on human health as well as on food security in temperate agricultural regions [32]. Aflatoxins are natural carcinogens that have the potential to suppress the immune system plus induces hepatocellular carcinoma, that is the cause of deaths in humans as well as livestock [33,34]. It is one of the most toxic among the four principal aflatoxins B1, B2, G1, and G2. International organizations carefully check the concentration of Aflatoxin in food and feed because of their noticeable impact on humans [35]. Lack of access to the markets, degradation in value results losses in the economy are the ultimatums of the Products affected by aflatoxins [34].

Aflatoxins are considered a critical indicator of the quality of the product, on the account of its carcinogenic disasters on human health. At the stages of pre-harvest and post-harvest, when the conditions are favorable for the mycotoxic species, maize gets affected by aflatoxins. It is possible that contamination appearance occurs along the distribution chain from the field to maize storage. Although, the critical risk point for aflatoxin contamination on the maize distribution chain is still not known. So, careful handling is necessary to identify high-risk points along the distribution channel. In this scrutiny, the risk of aflatoxin contamination was identified along the maize distribution chain, from field to the storage of maize. The results of the previous study were provided suggestions for maize enterprise actors to put interest in the handling of maize during storage after threshing, specifically to control the environmental storage conditions and time in order to lessen contamination by aflatoxin [36].

It was claimed that Aflatoxins were originated from the different regions of Africa, Asia and America. The maize crop found to be more vulnerable to the aflatoxins due to the presence of more moisture at the time of harvesting which favored the mold growth on cereals. In Democratic Republic of Congo, aflatoxin assessment in maize was done by using high-performance liquid chromatography. The assessment showed that $32 \%$ of the samples (out of a total of 50) were found positive, with aflatoxin levels $1.5-51.23 \mathrm{mg} / \mathrm{kg}$ for AFB1 and (3.1 - $103.89 \mathrm{mg} / \mathrm{Kg}$ ) for total aflatoxin [37].

\section{Health implications of aflatoxins}

Aflatoxins have been found to be highly hazardous to humans, animals and plants. Aflatoxin B1, which is produced by Aspergillus species, is classified among the most toxic compounds found in nature. This Aflatoxin is responsible for causing large-scale damage to various crops particularly rice, wheat, maize and peanuts. Furthermore, the consumption of these contaminated crops by humans and animals causes serious health implications and, in the worst case, may even lead to the death of the individuals [38].

The investigation on the poisonous effects of the aflatoxins started for the first time in history when it affected the poultry in turkey. It was observed that the Duckling is more vulnerable to the disease 
and the toxic effects produced by the aflatoxins as compared to the other animals. But several other animals such as chicken, rainbow trout, rabbits, dogs, monkeys and especially domesticated animals were also included among the affecters of aflatoxins [39].

Aflatoxins have been proved to be lethal for the domestic as well as experimental animals and these have also been suspected of possessing carcinogenic activity. The health implications were different in different animals and also varied with age, sex, species and hormonal status. In a study on mouse and duckling, it was observed that the mouse was more resistant than the duckling. Further studies showed that the animals deficient in protein were more susceptible to the aflatoxins but had the ability to stand against the carcinogenic effects of the aflatoxins [40].

The domestic animals on-farm secured contamination after eating food contaminated with the mycotoxins. This severe outbreak of disease affected many parts of Turkey. And, included among the effects were pigs, horses, poultry, dogs and camels. The human's beings, who consumed the mycotoxin contaminated food for longer periods, also suffered from the illness caused by these Mycotoxins. The diseases contracted by human beings owing to these deadly mycotoxins may include hepatitis B and C, weakening of the immune system, degeneration of testicles, and inflammation of different body parts [41].

In humans hepato-carcinogenesis the role of aflatoxins is complicated by hepatitis B virus infections. A number of epidemiological studies indicated that AFB1 was responsible for liver cancer and Kwashiorkor in humans. A positive relationship among levels of aflatoxins in the frequency of hepatocellular carcinoma and human diet was founded in many of the world countries such as Philippines, Swaziland, Kenya and Thailand [42].

Aflatoxins have been found responsible for causing Edema in malnourished adults and Kwashiorkor in malnourished children. Exposure of this contaminant in high doses may result in vomiting, abdominal pain, and even death in the worst case scenario, while in small quantities; it may lead to liver cancer [43].

\section{Detoxification of aflatoxins}

Worldwide scientists are continuously endeavoring to design a general and universal process of detoxification of aflatoxins, which can limit toxic substances to an acceptable level in various commodities. The series of events that led the discovery of aflatoxins as a potent carcinogen, mechanism of action, and its biosynthesis and structure function relationship provides interesting approach as cost-effective and technical factors which are involved in the development of an effective management plan for their detoxification [44].

Mycotoxin contamination of Wheat grains aimed for medium to long term storage could be reduced to satisfactory level by the adoption of various preventive measures such as, rapid drying of wheat grains, storing the wheat grains in clean, hygienic, and insects and microorganism's free warehouses, and regulating the moisture content of wheat grains properly [45].

Owing to carcinogenic effects and other multiple health hazards of these aflatoxins, a number of physical (mechanical sorting, thermal treatment or heat inactivation, irradiation and density segregation), chemical (treating with ammonia, sodium bisulfate and propionic acid) and biological (Streptococcus lactis and Bacillus subtilis) methods have been studied and applied for the management, detoxification and inactivation of AFs from contaminated food and feed commodities. In most cases, it is nearly impossible to prevent Mycotoxin contamination in cereals, but, various pre and post-harvest practices may reduce the risk of Mycotoxin contamination to the satisfactory level. This is especially important in preventing the exposure of humans to these harmful fungal metabolites and resultantly, warding off a great many diseases caused by these mycotoxins $[46,47]$.

Literature suggested that sodium hydrosulfite $\left(\mathrm{Na}_{2} \mathrm{~S}_{2} \mathrm{O}_{4}\right)$ could also be used for detoxification of aflatoxins. In an study conducted by Jalili., et al. [48], detoxification efficiency of sodium hydrosulfite was determined under high pressure (1.5bar) and high temperature $\left(121^{\circ} \mathrm{C}\right)$ for 15 seconds with different concentration $(0.25,0.5$, $1,1.5$ and $2 \%$ ). Results showed that maximum reduction occur at $2 \%$ with high pressure and heat.

Contamination of foods and feeds by aflatoxins has become a serious problem worldwide because these aflatoxins are able to pass through all the metabolic pathways unchanged and can also accumulate in the tissues of the body which ultimately pose serious threat to human and animal health [49].

However various detoxification techniques such as heat treatment, separation of contaminated seeds by mechanical methods, extraction by solvents as well as detoxification by chemical agents 
and added sorbents have been employed. However, no one seems capable to fulfill the safety, efficiency, preservation of nutritional elements and cost benefit ratio of any process regarding aflatoxins detoxification [50].

\section{Biological detoxification of aflatoxins}

Worldwide biological approaches are adapted for the degradation of pesticide residues and other toxins etc. in different agricultural commodities. One of the renowned strategies for aflatoxins management in feed and foodstuff is biodegradation by using microorganisms or enzymes. Since these methods of bio-detoxification are specific, efficient and environment friendly in eliminating the possible contamination of aflatoxins in foods and feeds, that's why, these are actively being assessed and can prove a promising choice. Fungi not only produce AFs but also are capable of degrading them. Four strains of fungi namely Eurotium herbarirorum, Aspergillus niger, Rhizopus spp., and non- aflatoxins producing species of $A$. flavus are able to transfer $\mathrm{AFB}_{1}$ to aflatoxicol (AFL) by reducing its cyclopentenon carbonyl. These fungal strains can also transfer $\mathrm{AFB}_{1}$ to aflatoxicol-A (AFL-A) then AFL-A can be transferred to aflatoxicol-B (AFL-B) through the actions of organic acids [51].

On the other hand, cells of the protozoon such as Tetrahymena pyriformis have the potential to degrade pure $\mathrm{AFB}_{1}$ to another bright florescent compound and decrease its concentration upto $25 \%$ in about 30 hours but, the way of $\mathrm{AFG}_{1}$ by this strain is different [51].

During a study [21] investigated and compared the aflatoxin degradation efficiency of a Rhodococcus pyridinivorans strain as bacterial inoculum and as bacterial extract in both the liquid and solid phases of an aflatoxin $\mathrm{B}_{1}$ contaminated bio-ethanol byproduct (i.e. whole stillage) during a three-day incubation period. Results indicate that 3 day microbial treatment is not capable of causing aflatoxin $\mathrm{B}_{1}$ degradation because of its tight binding into the solid stillage. That is why long, 12 day degradation experiment was conducted in which the degradation profile of this strain was found out. Between $3^{\text {rd }}$ and $7^{\text {th }}$ day of microbial reaction aflatoxin content was remarkably lowered down.

In another study, Adebo., et al. [22] conducted investigation on the biodegradation of aflatoxin $\mathrm{B}_{1}\left(\mathrm{AFB}_{1}\right)$, in this study, lysates of 3 bacterial strains (Pseudomonas anguilliseptica $\mathrm{VGF}_{1}$, Pseudomonas fluorescens and Staphylococcus sp. VGF ${ }_{2}$ ), which were separated from a gold mine aquifer, were used. To get protease free lysates, intermittent lysing of bacterial cells was done in the absence and presence of protease inhibitors. After this, these were incubated with Aflatoxin $\mathrm{AFB}_{1}$ for 3, 6, 12, 24 and 36 hours. High performance liquid chromatography (HPLC) was used to detect whether any $\mathrm{AFB}_{1}$ degradation occurred or not. HPLC results showed that lysates of Staphylococcus $s p$. were inhibited by protease after 6 hours of incubation period. $\mathrm{VGF}_{2}$ degraded the $\mathrm{AFB}_{1}$ by $100 \%$, while $P$ anguilliseptica $\mathrm{VGF}_{1}$ and $P$. fluorescens lysates performed $66.5 \%$ and $63 \%$ degradation of $\mathrm{AFB}_{1}$ respectively. None of the $\mathrm{AFB}_{1}$ was left to be degraded by any lysate after 12 hours of incubation.

\section{Conclusion}

The current review has been concluded that aflatoxins grow on agricultural food when the moisture cross the standard limit. The toxic materials are produced by aflatoxins which are directly affected human health. For the production of aflatoxin free food, detoxification and bio-detoxification technique is applied that can aid to eliminate aflatoxins in foodstuff.

\section{Bibliography}

1. Udomkun P., et al. "Innovative technologies to manage aflatoxins in foods and feeds and the profitability of application-A review". Food Control 76 (2017): 127-138.

2. Riba A., et al. "Aspergillus section Flavi and aflatoxins in Algerian wheat and derived products". Food and Chemical Toxicology 48.10 (2010): 2772-2777.

3. Kara GN., et al. "Co-occurrence of aflatoxins and ochratoxin A in cereal flours commercialised in Turkey". Food Control 54 (2015): 275-281.

4. Wacoo AP., et al. "Methods for detection of aflatoxins in agricultural food crops". Journal Applied Chemistry (2014): 706291.

5. Dors GC., et al. "Aflatoxins: contamination, analysis and control". Embrapa Suínos e Aves-Capítulo em livro científico (ALICE) (2011).

6. Catanante G., et al. "An overview of recent electrochemical immunosensing strategies for mycotoxins detection". Electroanalysis 28.8 (2016): 1750-1763.

7. Hussein HS., et al. "Toxicity, metabolism, and impact of mycotoxins on humans and animals". Toxicology 167.2 (2001): 101134. 
8. Womack ED., et al. "A recent review of non-biological remediation of aflatoxin-contaminated crops". Journal of the Science of Food and Agriculture 94.4 (2014): 1706-1714.

9. Stroka J and Anklam E. "New strategies for the screening and determination of aflatoxins and the detection of aflatoxinproducing moulds in food and feed". TrAC Trends in Analytical Chemistry 21.2 (2002): 90-95.

10. Asghar MA., et al. "Occurrence of aflatoxins contamination in brown rice from Pakistan". Iranian journal of Public Health 43.3 (2014): 291.

11. Iqbal SZ., et al. "Detection of aflatoxins and zearalenone contamination in wheat derived products". Food Control 35.1 (2014): 223-226.

12. Beizaei A., et al. "Highly sensitive toxin microarray assay for aflatoxin B1 detection in cereals". Food Control 57 (2015): 210-215.

13. Liu Z., et al. "Aflatoxins in stored maize and rice grains in Liaoning Province, China". Journal of stored Products Research 42.4 (2006): 468-479.

14. Prietto L., et al. "Post-harvest operations and aflatoxin levels in rice (Oryza sativa)”. Crop Protection 78 (2015): 172-177.

15. Rustom IY. "Aflatoxin in food and feed: occurrence, legislation and inactivation by physical methods". Food Chemistry 59.1 (1997): 57-67.

16. Shanakht H., et al. "Characterization of Fungal Microbiota on Rice Grains From Local Markets of Lahore". Journal of Hygienic Engineering and Design 9 (2014): 35-40.

17. Bryden WL. "Mycotoxins in the food chain: human health implications". Asia Pacific Journal of Clinical Nutrition 16.S1 (2007): 95-101.

18. Matumba L., et al. "Fungal metabolites diversity in maize and associated human dietary exposures relate to micro-climatic patterns in Malawi”.World Mycotoxin Journal 8.3 (2014): 269282.

19. Lutfullah $\mathrm{G}$ and A Hussain. "Studies on contamination level of aflatoxins in some cereals and beans of Pakistan". Food Control 23.1 (2012): 32-36.

20. Creppy EE. "Update of survey, regulation and toxic effects of mycotoxins in Europe”. Toxicology letters 127.1 (2002): 19-28.
21. Prettl Z., et al. "Biological degradation of aflatoxin B 1 by a Rhodococcus pyridinivorans strain in by-product of bioethanol". Animal Feed Science and Technology 224 (2017): 104-114.

22. Adebo OA., et al. "Aflatoxin B 1 degradation by liquid cultures and lysates of three bacterial strains". International Journal of Food Microbiology 233 (2016): 11-19.

23. Ramesh J., et al. "Survey of market samples of food grains and grain flour for Aflatoxin B1 contamination". International Journal Current Microbiological Applied Sciences 2.5 (2013): 184188.

24. Sabino M., et al".Variations in the levels of aflatoxin in cows milk consumed in the city of São Paulo, Brazil". Food Additives and Contaminants 6.3 (1989): 321-326.

25. McKevith Brigid. "Nutritional aspects of cereals". Nutrition Bulletin 29.2 (2004): 111-142.

26. Zhang $\mathrm{H}$ and B Wang. "Fate of deoxynivalenol and deoxynivalenol-3-glucoside during wheat milling and Chinese steamed bread processing". Food Control 44 (2014): 86-91.

27. Elzupir Amin 0., et al. "The occurrence of aflatoxin in rice worldwide: a review". Toxin Reviews 34.1 (2015): 37-42.

28. Mazaheri M. "Determination of aflatoxins in imported rice to Iran". Food and Chemical Toxicology 47.8 (2009): 2064-2066.

29. Iqbal SZ., et al. "The presence of aflatoxins and ochratoxin A in rice and rice products; and evaluation of dietary intake". Food Chemistry 210 (2016): 135-140.

30. Khalid W., et al. "Assessment of aflatoxins in wheat flour from different areas of Punjab in Pakistan". International Journal of Biosciences 17.3 (2020): 230-240.

31. Razdari HB., et al. "An Assessment of Aflatoxin Levels in Wheat Samples of 5 top Provinces of Iran" (2014).

32. Bandyopadhyay Ranajit., et al. "Biological control of aflatoxins in Africa: current status and potential challenges in the face of climate change". World Mycotoxin Journal 9.5 (2016): 771-789.

33. Liu Yan and Felicia Wu. "Global burden of aflatoxin-induced hepatocellular carcinoma: a risk assessment". Environmental Health Perspectives 118.6 (2010): 818-824.

34. Wu Fang. "Global impacts of aflatoxin in maize: trade and human health". World Mycotoxin Journal 8.2 (2015): 137-142. 
35. Machida M andGomi K. "Aspergillus: molecular biology and genomics". Horizon Scientific Press (2010).

36. Marpaung, David Septian Sumanto., et al. "Determination of Aflatoxin Contamination Risk along Maize Distribution Chain (Case study: A Maize Enterprise in East Lampung)". IOP Conference Series: Earth and Environmental Science 537.1 (2020).

37. Kamika I and M Tekere. "Occurrence of aflatoxin contamination in maize throughout the supply chain in the Democratic Republic of Congo". Food Control 69 (2016): 292-296.

38. Phillips TD., et al. "Reducing human exposure to aflatoxin through the use of clay: a review". Food Additives and Contaminants 25.2 (2008): 134-145.

39. Yunus Agha W., et al. "Aflatoxin B1 in affecting broiler's performance, immunity, and gastrointestinal tract: A review of history and contemporary issues". Toxins 3.6 (2011): 566-590.

40. Benkerroum Noreddine. "Chronic and acute toxicities of aflatoxins: mechanisms of action". International Journal of Environmental Research and Public Health 17.2 (2020): 423.

41. Ali SW and S Afzaal. "Aflatoxins in Pakistani Foods: A Serious Threat to Food Safety". Journal of Hygienic Engineering and Design 9 (2014): 20-25.

42. Massey TE., et al. "Biochemical and molecular aspects of mammalian susceptibility to aflatoxin B1 carcinogenicity". Proceedings of the Society for Experimental Biology and Medicine 208.3 (1995): 213-227.

43. Van Rensburg S., et al. "Hepatocellular carcinoma and dietary aflatoxin in Mozambique and Transkei". British Journal of Cancer 51.5 (1985): 713-726.

44. Mishra H and C Das. "A review on biological control and metabolism of aflatoxin" (2013).

45. Lemmens Marc., et al. "The effect of inoculation treatment and long-term application of moisture on Fusarium head blight symptoms and deoxynivalenol contamination in wheat grains". European Journal of Plant Pathology 110.3 (2004): 299-308.

46. Aliabadi MA., et al. "Biological control of aflatoxins". European Journal of Experimental Biology 3.2 (2013): 162-166.
47. Velazhahan R. et al. "Detoxification of aflatoxins by seed extracts of the medicinal plant, Trachyspermum ammi (L.) Sprague ex Turrill-structural analysis and biological toxicity of degradation product of aflatoxin G1". Food Control 21.5 (2010): 719-725.

48. Jalili M and S Jinap. "Role of sodium hydrosulphite and pressure on the reduction of aflatoxins and ochratoxin A in black pepper". Food Control 27.1 (2012): 11-15.

49. Anthony Makun Hussaini., et al. "Aflatoxin contamination in foods and feeds: A special focus on Africa". Trends in Vital Food and Control Engineering 187 (2012).

50. Karlovsky Petr., et al. "Impact of food processing and detoxification treatments on mycotoxin contamination". Mycotoxin research 32.4 (2016): 179-205.

51. Wu Qinghua., et al. "Biological degradation of aflatoxins". Drug Metabolism Reviews 41.1 (2009): 1-7.

\section{Assets from publication with us}

- Prompt Acknowledgement after receiving the article

- Thorough Double blinded peer review

- Rapid Publication

- Issue of Publication Certificate

- High visibility of your Published work

Website: https://www.actascientific.com/

Submit Article: https://www.actascientific.com/submission.php Email us: editor@actascientific.com

Contact us: +919182824667 